\title{
Decreasing Number of Food Portions Consumed by an Adolescent Female with Autism
}

\author{
Gabriela A. Chagolla ${ }^{1} \cdot$ Becky Penrod $^{1}$ (i) \\ Accepted: 24 August 2021 / Published online: 27 September 2021 \\ (C) Association for Behavior Analysis International 2021
}

\begin{abstract}
The current study evaluated the effectiveness of a treatment package including a behavior contract, brief portion selection training, and a food diary, to manage portion control in an adolescent female with autism. The behavior contract specified a reinforcement contingency for meeting a weekly goal that described how many servings the participant may consume but should not exceed during meals consisting of target foods. Results of the study demonstrated that the treatment package was successful in managing the number of portions the participant consumed across treatment and generalization sessions. $\bullet$ The flexible nature of the intervention may lead to greater treatment adherence $\bullet$ The intervention was rated with high social acceptability. $\bullet$ The intervention is generalizable to other naturalistic contexts. $\bullet$ The intervention may promote independence through teaching selfmanagement skills.
\end{abstract}

Keywords behavior contract $\cdot$ portion control $\cdot$ autism

A behavior contract is a written agreement between two or more people that specifies a contingent relationship between the occurrence (or nonoccurrence) of a target behavior and access to a specified reward (Cooper et al., 2019; Miltenberger, 2016). Key components of behavior contracts include clearly stated expectations of the behavior targeted for change (Bowman-Perrott et al., 2015), a description of how the target behavior will be measured, and a description of reinforcement or punishment contingencies (Miltenberger, 2016).

Behavior contracts have several advantages. They are cost effective and can be implemented as part of a packaged intervention (Bowman-Perrott et al., 2015). Further, behavior contracts can easily be used by a large range of practitioners (Cooper et al., 2019) including teachers, school psychologists, and educators (Bowman-Perrott et al., 2015). Behavior contracts can be used to target a wide variety of behaviors across settings. For example, behavior contracts have been used in

This research was completed in partial fulfillment of thesis requirements by the first author. We thank the families for participating in our research.

Becky Penrod

Penrodb@csus.edu

1 Department of Psychology, California State University, Sacramento, 6000 J Street, Sacramento, CA 95819-6007, USA classroom settings to teach students with developmental disabilities how to adhere to classroom rules (e.g., Mruzek et al., 2007) or engage in social interactions with their peers (e.g., Alwahbi \& Hua, 2020). Behavior contracts have also been used to improve or correct performance in contexts that vary from higher education to prison settings (Bowman-Perrott et al., 2015).

Behavior contracts have been demonstrated to improve a wide range of outcomes (Bowman-Perrott et al., 2015). In a meta-analysis of single-case research on behavior contracts, Bowman-Perrott et al. (2015) concluded that students across age groups, gender, and disability status benefit from contracts, both in terms of academic outcomes and reduction of inappropriate behaviors. Relevant to the current study, behavior contracts have also been documented to produce gains in health-related behaviors such as treatment adherence to exercise programs (e.g., Washington et al., 2014). Although individuals with disabilities, including autism spectrum disorder (ASD), have participated in several studies evaluating the effectiveness of behavior contracts (Bowman-Perrott et al., 2015), to our knowledge behavior contracts have not been used to specifically address health related behaviors of children with ASD. We entered relevant key terms (i.e., behavior contract, contingency contract, goal setting, autism, ASD, portion control, serving) into a library database (PsycINFO) and also reviewed table of contents of multiple behavior analytic 
journals (e.g., JABA, BAP, Behavior Modification) in the last 5 years. Results of this search did not identify any studies that specifically used a behavior contract to target health related behaviors of children with ASD.

In summary, there is precedence for the use of behavior contracts to promote health related behavior as well as research demonstrating their effective use among children with ASD when combined with other treatment components. Thus, the aim of the current study was to pilot the use of a behavior contract supplemented with a brief portion selection training and a food diary to decrease the number of portions consumed by an adolescent female with ASD.

\section{Method}

\section{Participants, Setting, and Materials}

"Lea", was a 17-year-old female diagnosed with ASD. At the time of the study, Lea had a body mass index (BMI) of 26.8, which put her in the $89^{\text {th }}$ percentile for a female her age. Lea had an extensive vocal verbal repertoire: she spoke in full sentences and demonstrated comprehension of verbal rules by answering questions related to instructions given by experimenters. However, no formal measure of her verbal repertoire was conducted.

Lea previously participated in a study that aimed to reduce the pace at which she ate a meal (see Page et al., 2017). Although Lea's parents reported they were happy with the progress Lea had made, her mother expressed concern that Lea "seems to have no sensory input on satiety until she is overly full." In consequence, Lea's mother reported that she often restricts access to snack foods. Lea's mother also noted that Lea often wakes up in the middle of the night to snack, possibly as a result of access to snack foods being restricted. Lea's mother expressed that she would like to give Lea more control over what she eats and when, to work toward independence as Lea gets older.

Sessions were conducted in the family's home in the dining area, which included a dinner table with two benches and place settings. Lea and the experimenter were seated at the table and ate dinner together. Sessions were arranged in this manner to simulate a situation in which Lea would be required to manage the number of portions she consumes without a parent there to prompt her (e.g., living with a roommate). Additional materials included a food diary, data sheets, pens, a food scale, a timer, and a GoPro video recording device.

\section{Experimental Design and Dependent Measures}

A changing criterion embedded within a concurrent multiple probe design across foods was used to evaluate the effects of the intervention. In all conditions, data on number of servings consumed of the primary target food was collected. A serving of the target food was determined by the amount of food specified as a serving on the nutrition label or in the nutritional facts. For example, one serving of a large cheese pizza is one slice. Data on the percentage of the serving consumed of the secondary food was also collected. In particular, the experimenter weighed the food in grams at the start and end of the meal. The experimenter subtracted the postmeal weight from the premeal weight and the resulting number was then divided by the premeal weight and multiplied by 100 to calculate a percentage. If the plate was empty at the end of the session, the experimenter did not weigh the plate again and recorded the percentage of the secondary food consumed as $100 \%$.

\section{Interobserver Agreement and Treatment Integrity}

A secondary, independent observer collected interobserver agreement (IOA). The total agreement method was used to calculate IOA on all measures by dividing the smaller recorded number by the larger and then converting the output to a percentage. IOA was calculated for the number of servings consumed for $71 \%$ of sessions and mean agreement was $100 \%$. IOA for percentage of the portion of secondary food consumed was calculated for $29 \%$ of sessions, with a mean of 99.8\% (range: $98 \%-100 \%$ ) agreement.

During treatment sessions, treatment integrity (TI) data were collected on whether the experimenter had additional servings of the target and secondary food present, refrained from providing input regarding the number of servings consumed and information Lea was recording in her food diary, and ended the session at the specified time. TI data were calculated by dividing the number of correct steps by the total number of steps and then converting the output to a percentage; percentages were then averaged across sessions. TI data were collected for $83 \%$ of all sessions, with a mean of $99 \%$ (range: $80 \%-100 \%$ ) correct implementation. Further, information on whether the reinforcer was delivered or not was gathered via parental report at the next session after the reinforcer was to be delivered. Parents occasionally reported they had to delay the delivery of the reinforcer by a day or two due to competing activities. On one occasion, there was an error of omission in which parents reported they forgot to deliver the reinforcer until the next scheduled session which was 5 days after the scheduled delivery. On this occasion, experimenters reminded parents of the importance of delivering the reinforcer at the scheduled time.

\section{Experimental Procedures}

\section{Food Selection}

Target foods included a large cheese pizza and a can of chips, selected from a list of food mother reported to be problematic. Each target food was paired with a second complementary 
food. Pizza was paired with salad (i.e., lettuce, tomatoes, ranch, and croutons) and chips was paired with a turkey and cheese sandwich (i.e., two slices of bread, turkey, and cheese). A secondary food was included to simulate a typical meal; that is, parents reported they typically offered salad with pizza and turkey sandwiches with chips. We were also interested in measuring whether Lea would consume more of the complementary food as she consumed less of the target food.

\section{Baseline}

During every baseline session, Lea was presented with an entire large cheese pizza and a bowl of salad or an entire can of chips and a turkey and cheese sandwich. Lea was given free access to consume as much or as little of the target foods without any input from the experimenter. For all experimental sessions, Lea ate the targeted foods, and the experimenter ate their own meal that they brought from home. Lea's food was weighed at the start of each meal to ensure the amount of food presented remained constant across sessions. Meals were terminated once Lea verbally indicated she was finished eating her meal, if no food was remaining (i.e., Lea consumed all food presented), or after $1 \mathrm{hr}$ elapsed, whichever occurred first. Sessions were conducted twice per week during Lea's dinner time.

\section{Brief Portion Selection Training}

Prior to treatment, the experimenter provided a brief portion selection training. No assessment regarding Lea's knowledge on this topic was conducted prior to the training. The training was based on information from The Food Guide Pyramid(U.S. Department of Agriculture, 1992) and "The Fun of Pizza, the Balance of Good Nutrition: Grains, Dairy, Protein and Vegetables by the Slice" (Young, 2013). Information was presented verbally with the use of visuals, including appropriate serving sizes and number, and healthy food choices. Lea was asked comprehension questions to ensure she was attending to the information (e.g., "What is a serving?," "Where can you find how much a serving of your favorite food is?"). If Lea did not answer correctly, the experimenter prompted her through the correct response by referring back to the visuals. Once Lea verbally indicated she understood each question (i.e., Lea answered the question correctly the first time or after referring back to the visuals), the brief training was considered complete. It took approximately $10 \mathrm{~min}$ to review all of the information, which was provided to Lea only one time after baseline.

\section{Treatment Package (Behavior Contract + Food Diary)}

Treatment sessions were conducted similar to baseline conditions with respect to how meals were presented and terminated. However, during the treatment condition, a behavior contract was in place and a food diary was used as a means of data collection such that Lea could self-manage her portions. At the start of the week (considered the 1st day after the end of the previous contract during which a session took place), the experimenter went over a behavior contract with Lea. The behavior contract specified the start and end date of the week; the goal for each target food; and a "special treat" Lea could earn at the end of the week if she met her weekly goal (i.e., "If I meet my weekly goal, then I will get a special treat of [item] on [day]"). If Lea did not meet her goal, the contract specified she could try again the following week (i.e., "If I do not meet my weekly goal, I will not receive my special treat and I can try again next week").

The experimenter and Lea determined the maximum number of servings Lea was allowed to consume, but should not exceed, in order to earn access to a reinforcer (i.e., special treat), decided by Lea and the experimenter, with parent approval. The special treat was chosen as the reinforcer in an effort to teach Lea to be planful and eat less healthy food options in moderation, similar to adults who may treat themselves to a "cheat" food once per week. The behavior contract was signed by Lea, the parent, and the experimenter after all components were agreed upon. The experimenter determined if Lea's behavior met criterion for reinforcement and then instructed parents to provide the special treat at the end of the week that was specified on the behavior contract. Based on parent report, Lea consumed the reinforcer at the same time parents purchased it for her.

During treatment sessions, a food diary was used such that Lea could self-manage her portions. Prior to treatment, the experimenter explained how to use the food diary by filling out an example page for Lea to reference and going over each piece of information to be recorded, including: (1) type of food consumed (e.g., pizza and salad), (2) number of servings of the target food consumed, and (3) whether she met her goal for that session. At the end of the week, Lea also recorded whether she met her weekly goal. Lea was not provided with any instruction regarding when during the session she should record the information, nor feedback regarding what information was recorded. However, if Lea only partially filled out her diary, the experimenter reminded her to complete the diary at the end of the meal.

\section{Generalization Sessions}

Lea's parents reported that in a family meal context, Lea often attempts to eat more than her share of food. Thus, three generalization probes with pizza were conducted to determine if Lea would continue to adhere to her goal in the context of sharing a meal with others. The behavior contract was in place across all probe sessions. The first generalization probe was conducted with Lea and the experimenter in which they shared one pizza. The second and third generalization probes were conducted with Lea and her parents and Lea and her family (i.e., parents and siblings), respectively. 


\section{Social Validity}

Social validity questionnaires were completed at the conclusion of the study. The parent questionnaire consisted of 14 questions modified from Hoch et al. (1994). Lea's social validity questionnaire consisted of four items modified from Hood et al. (2019).

\section{Results}

During baseline, Lea consumed an average of 5.67 (range: 56) servings of pizza. When the treatment package was implemented, number of servings consumed matched the criterion in place throughout all phases of the intervention, starting with an initial goal of five servings, which decreased to three servings. Following the third treatment phase, a bidirectional shift in criterion was implemented. The criterion was increased to four during sessions 21 and 22, and Lea consumed 4 and 3.58 servings, respectively, further demonstrating a functional relationship between the treatment package and the number of servings consumed. In the last phase, the criterion was 3 servings and Lea consumed a mean of 3.16 servings (range: 3-4). Due to the COVID-19 pandemic, there was a 2-month gap between sessions 30 and 31 . When sessions resumed, sessions 31 and 32 were conducted via Zoom. During generalization sessions, Lea continued to consume the criterion number of three servings while sharing pizza with others.

For the second target food, chips, Lea consumed an average of five servings during baseline. When the treatment package was implemented, an initial goal of 4 servings was agreed upon and Lea consumed a mean of 3.8 (range: 3.21-4) servings. During the second phase, the criterion was 3 servings and Lea consumed a mean of 2.96 (range: 2.79-3) servings. Data were also collected on the accuracy with which Lea recorded number of servings consumed. Percentage of sessions in which Lea accurately recorded number of servings was $93 \%$.

With respect to the secondary foods, the mean percentage of the portion consumed across all sessions was $63 \%$ (range: 20\%-100\%) for salad and 66\% (range: $24 \%-100 \%$ ) for turkey sandwich. An inverse relationship between the target food and secondary food was not observed. However, it should be noted there were two sessions in which baseline data for salad were not collected due to experimenter error (Fig. 1).

Social validity data indicated high acceptability of the procedure from both the parent and the participant. On a 5-point Likert scale, Lea's parent rated a 5 (strongly agree) for being satisfied with the services their child received, a 5 for their interactions with the treatment team, and a 4.2 (range: 4-5) for improvement of other skills or their child's health.

On a 7-point Likert scale, Lea rated a 7 (strongly agree) and 6 (agree) for being satisfied with the strategies taught to monitor the number of servings consumed and eat healthier alternatives. She rated a 6 for being satisfied with the procedures used to teach portion control and a 5 (somewhat agree) for recommending this procedure to other individuals her age who want to work on this skill.

\section{Discussion}

Overall, results demonstrate how a behavior contract can easily be created to address a socially significant problem that behavior analysts may face in their clinical work. In addition, this intervention demonstrated how a behavior contract may be supplemented with additional components to increase its efficacy. In previous studies, behavior contracts with children with ASD have been evaluated in conjunction with other treatment components including behavior prompts (e.g., Alwahbi \& Hua, 2020) and stimulus prompts (e.g., Mruzek et al., 2007). In the current study, the self-monitoring component consisted of recording information in a physical notebook. Although Lea was not provided with any instructions regarding when during the session she should record the information, Lea kept the notebook at the dining table and opted to fill out the diary prior to or during the meal. Thus, the food diary may have functioned as a stimulus prompt, which could have contributed to the effectiveness of the behavior contract. However, the use of a physical notebook may not be the most pragmatic for young adults. Thus, future research should evaluate the use of a food tracking app, which would provide participants with a discrete way to monitor their behavior, in particular in social contexts. Future research should also continue to evaluate additional treatment components that may contribute to the success of behavior contracts with children with ASD. Such components may include having the contract visible during the meal, recording the number of servings consumed directly on the contract, or reviewing the contract before every meal rather than at the start of the week.

Both Lea and her parents rated the intervention highly. It should be noted that allowing Lea to negotiate her goals may have contributed to the high social validity rating, which supports previous recommendations that all parties negotiate the terms of the behavior contract to ensure it is acceptable to everyone involved (Miltenberger, 2016). When the first behavior contract goal was being negotiated, Lea stated the goal was not attainable. Therefore, the experimenter modified it to a goal that Lea found acceptable. By having the client 


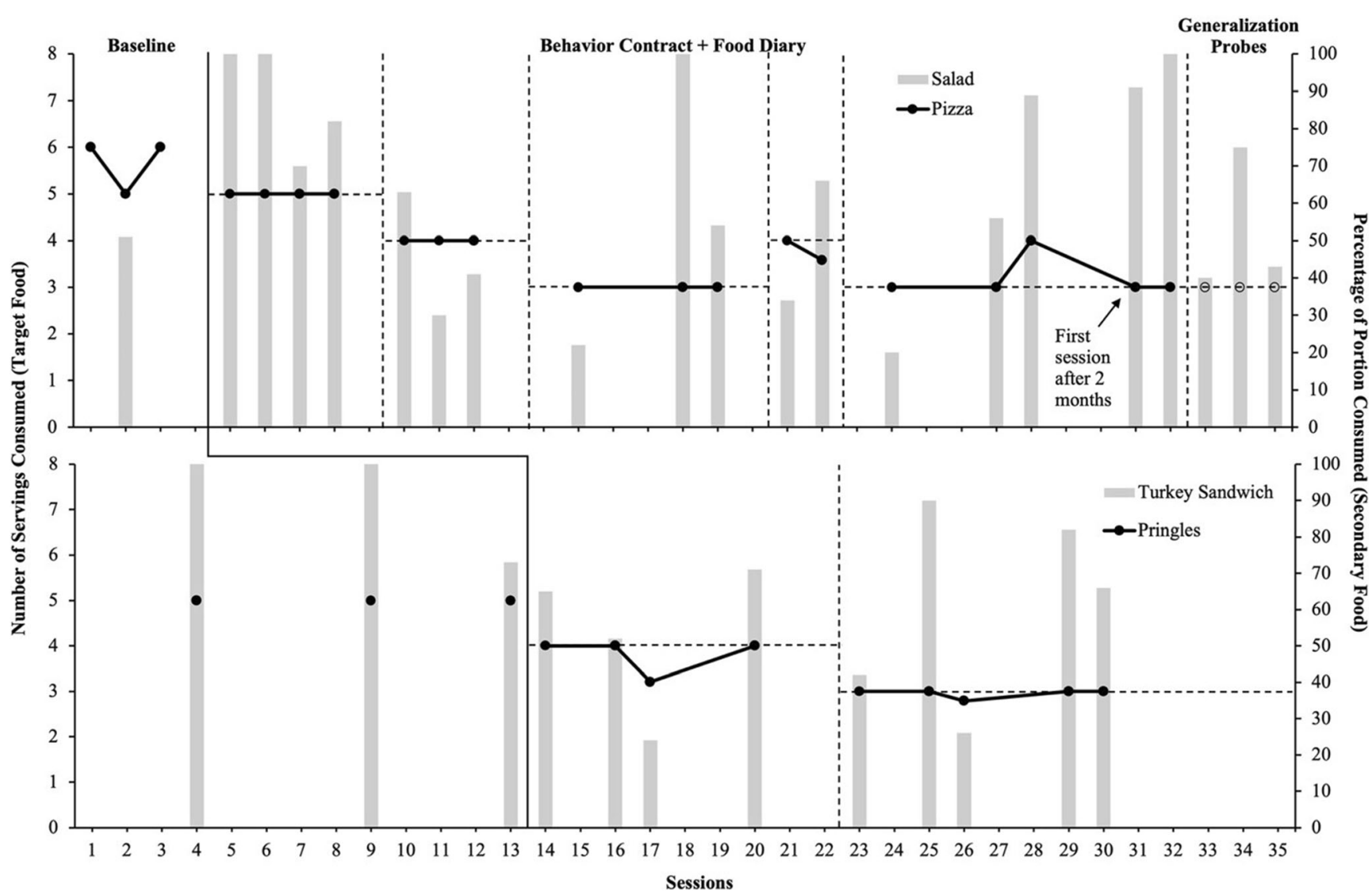

Fig. 1 Number of servings of the target food consumed is depicted along the primary $y$-axis and the percentage of portion consumed of the secondary food is depicted by gray bars along the secondary y-axis. Note.
Criterion shifts are depicted by the dashed horizontal lines. A brief portion selection training was conducted prior to the start of session number 5

Funding None

Availability of Data and Material Available upon request.

\section{Declarations}

Ethics Approval This study was approved by the institutional review board of California State University, Sacramento.

Consent to Participate The participant consented to participate in the study.

Conflict of Interest We declare no known conflicts of interest.

\section{References}

Alwahbi, A., \& Hua, Y. (2021). Using contingency contracting to promote social interactions among students with ASD and their peers. Behavior Modification, 45(5),671-694. https://doi.org/10.1177/ 0145445520901674

Bowman-Perrott, L., Burke, M. D., de Marin, S., Zhang, N., \& Davis, H. (2015). A meta-analysis of single-case research on behavior 
contracts: effects on behavioral and academic outcomes among children and youth. Behavior Modification, 39, 247-269. https://doi. org/10.1177/0145445514551383

Cooper, J. O., Heron, T. E., \& Heward, W. L. (2019). Applied behavior analysis (3rd ed.). Pearson Education.

Hoch, T. A., Babbitt, R. L., Coe, D. A., Krell, D. M., \& Hackbert, L. (1994). Contingency contacting: Combining positive reinforcement and escape extinction procedures to treat persistent food refusal. Behavior Modification, 18, 106-128. https://doi.org/10.1177/ 01454455940181007

Hood, S., Olsen, A., Luczynski, K., \& Randle, F. (2019). Improving accepting and giving compliments with individuals with developmental disabilities. Journal of Applied Behavior Analysis, 53, 10131028. https://doi.org/10.1002/jaba.662

Miltenberger, R. G. (2016). Behavior modification: Principles and procedures (6th ed.). Cengage Learning.

Mruzek, D., Cohen, W., \& Smith, C. (2007). Contingency contracting with students with autism spectrum disorders in a public school setting. Journal of Developmental \& Physical Disabilities, 19, 103-114. https://doi.org/10.1007/s10882-007-9036-x
Page, S. V., Griffith, K., \& Penrod, B. (2017). Reduction of rapid eating in an adolescent female with autism. Behavior Analysis in Practice, 10, 87-91. https://doi.org/10.1007/s40617-016-0143-y

U.S. Department of Agriculture. (1992). The food guide pyramid. https:// fns-prod.azureedge.net/sites/default/files/archived_projects/ FGPPamphlet.pdf

Washington, W. D., Banna, K. M., \& Gibson, A. L. (2014). Preliminary efficacy of prize-based contingency management to increase activity levels in healthy adults. Journal of Applied Behavior Analysis, 47(2), 231-245. https://doi.org/10.1002/jaba.119

Young, L. R. (2013). The fun of pizza, the balance of good nutrition: Grains, dairy, protein and vegetables by the slice. Nestle $S$. A.https:// www.nestleusa.com/sites/g/files/pydnoa536/files/asset-library/ documents/nutritionhealthwellness/pizza/pizzaportionguide_full. pdf

Publisher's Note Springer Nature remains neutral with regard to jurisdictional claims in published maps and institutional affiliations. 